Pacific Journal of Mathematics

FACTORIZATION OF POLYNOMIALS OVER FINITE FIELDS 


\title{
FACTORIZATION OF POLYNOMIALS OVER FINITE FIELDS
}

\author{
RICHARD G. SWAN
}

Dickson [1, Ch. V, Th. 38] has given an interesting necessary condition for a polynomial over a finite field of odd characteristic to be irreducible. In Theorem 1 below, I will give a generalization of this result which can also be applied to fields of characteristic 2 . It also applies to reducible polynomials and gives the number of irreducible factors mod 2 .

Applying the theorem to the polynomial $x^{p}-1$ gives a simple proof of the quadratic reciprocity theorem. Since there is some interest in trinomial equations over finite fields, e.g. [2], [4], I will also apply the theorem to trinomials and so determine the parity of the number of irreducible factors.

1. The discriminant. If $f(x)$ is a polynomial over a field $F$, the discriminant of $f(x)$ is defined to be $D(f)=\delta(f)^{2}$ with

$$
\delta(f)=\prod_{i<j}\left(\alpha_{i}-\alpha_{j}\right)
$$

where $\alpha_{1}, \cdots, \alpha_{n}$ are the roots of $f(x)$ (counted with multiplicity) in some extension field of $F$. Clearly $D(f)=0$ if $f$ has any repeated root. Since $D(f)$ is a symmetric function in the roots of $f, D(f) \in F$. An alternative formula for $D(f)$ which is sometimes useful may be obtained as follows:

$$
D(f)=\prod_{i<j}\left(\alpha_{i}-\alpha_{j}\right)^{2}=(-1)^{n(n-1) / 2} \prod_{i \neq j}\left(\alpha_{i}-\alpha_{j}\right)=(-1)^{n(n-1) / 2} \prod_{i} f^{\prime}\left(\alpha_{i}\right)
$$

where $n$ is the degree of $f(x)$ and $f^{\prime}(x)$ the derivative of $f(x)$. In $\S 4$, I will give still another way to calculate $D(f)$.

If $f(x)$ is monic with integral coefficients in some p-adic or algebraic number field, all $\alpha_{i}$ are integral and so $D(f)$ is integral. Consider the expression

$$
\delta_{1}=\prod_{i<j}\left(\alpha_{i}+\alpha_{j}\right) .
$$

This is integral and lies in $F$, being a symmetric function of the roots. Clearly $\delta(f)=\delta_{1}+2 \delta_{2}$ where $\delta_{2}$ is integral. Thus $D(f)=\delta(f)^{2} \equiv \delta_{1}^{2} \bmod$ 4 , so $D(f)$ is congruent to a square in $F \bmod 4$. This is a special case of a well-known theorem of Stickelberger [3, Ch. 10, Sec. 3].

Received November 15, 1961. The author is an Alfred P. Sloan Fellow.

Added in Proof. I have recently discovered that Theorem 1 of this paper is due to L. Stickelberger, Über eine neue Eigenschaft der Diskriminanten algebraischer Zahlkörper, Verh. 1 Internat. Math. Kongresses, Zurich 1897, Leipzig 1898, 182-193. A simplified proof, essentially the same as mine, was given by K. Dalen, On a theorem of Stickelberger, Math. Scand. 3 (1955), 124-126.

The applications of the theorem, however, seem to be new. 
Suppose $f(x)$ is monic with integral coefficients in a p-adic field $F$. I will denote by $\bar{f}(x)$ the polynomial over the residue class field obtained by reducing all coefficients of $F \bmod \mathfrak{p}$. In some extension field of $F^{*}$ we have $f(x)=\left(x-\alpha_{1}\right) \cdots\left(x-\alpha_{n}\right)$. Therefore $\bar{f}(x)=\left(x-\bar{\alpha}_{1}\right) \cdots(x$ $-\bar{\alpha}_{n}$ ) where $\bar{\alpha}_{i}$ is $\alpha_{i}$ reduced modulo the (unique) extension of $\mathfrak{p}$. It. follows that $D(\bar{f})$ is $D(f)$ reduced $\bmod \mathfrak{p}$. In particular, if $\bar{f}(x)$ has. no repeated root, $D(\bar{f}) \neq 0$ and so $D(f)$ is prime to $\mathfrak{p}$.

2. The main theorem. If $f(x)$ is a monic polynomial with integral coefficients in a $p$-adic field, I will again denote by $\bar{f}(x)$ the result of reducing the coefficients of $f(x) \bmod \mathfrak{p}$.

THeOREM 1. Let $f(x)$ be a monic polynomial of degree $n$ with. integral coefficients in a p-adic field $F$. Assume that $\bar{f}(x)$ has no repeated roots. Let $r$ be the number of irreducible factors of $\bar{f}(x)$ over the residue class field. Then $r \equiv n \bmod 2$ if and only if $D(f)$ is a. square in $F$.

Proof. Over the residue class field $K$ we can factor $\bar{f}(x)=\bar{f}_{1}(x)$ $\cdots \bar{f}_{r}(x)$. Since the $\bar{f}_{i}(x)$ are relatively prime, Hensel's lemma shows that there is a corresponding factorization $f(x)=f_{1}(x) \cdots f_{r}(r)$ over $F^{r}$ where $\bar{f}_{i}(x)$ is $f_{i}(x) \bmod \mathfrak{p}$. Since $\bar{f}_{i}(x)$ is irreducible over $K, f_{i}(x)$ is irreducible over $F$. Since $\bar{f}_{i}(x)$ has no repeated root, $D\left(f_{i}\right)$ is prime to $\mathscr{Y}$. Therefore the field $E_{i}$ obtained by adjoining a root of $f_{i}(x)$ to $F^{r}$ is unramified over $F$. Since there is only one unramified extension of $F$ of any given degree and that extension is cyclic, $E_{i}$ is cyclic over $F^{\prime}$ and thus is the splitting field of $f_{i}$. The splitting field $E$ of $f(x)$ is the composite of all the $E_{i}$ and therefore is unramified over $F$. Thus $E / F^{\prime}$ is a cyclic extension. (A more elementary proof of this was pointed out by W. Feit. Let $m$ be the least common multiple of the degrees of the $f_{i}(x)$. It is easy to construct a cyclic unramified extension $E_{1} / F^{r}$ of degree $m$ by adjoining a root of unity to $F$. Now $\bar{f}(x)$ splits completely over the residue class field $K_{1}$ of $E_{1}$ since the degree $n_{i}$ of every irreducible factor of $\bar{f}(x)$ divides $m=\left[E_{1}: F\right]$. By Hensel's lemma, $f(x)$, splits completely over $E_{1}$ so $E \subset E_{1}$.)

Let $\sigma$ generate the galois group of $E / F$. Let $\beta_{j}$ be a root of $f_{j}(x)$. Then the roots to $f_{j}(x)$ are given by $\sigma^{i}\left(\beta_{j}\right)$ for $0 \leqq i \leqq n_{j}-1$ where $n_{j}$ is the degree of $f_{j}(x)$. Thus the roots of $f(x)$ are $\sigma^{i}\left(\beta_{j}\right)$ for $j=1, \cdots$, $n, i=0, \cdots, n_{j}-1$. These can be ordered by defining $\left(i_{1}, j_{1}\right)<\left(i_{2}, j_{2}\right)$ if $j_{1}<j_{2}$ or if $j_{1}=j_{2}$ and $i_{1}<i_{2}$. For any $i$, the symbol $(i, j)$ will be interpreted to mean $\left(i^{\prime}, j\right)$ where $i^{\prime} \equiv i \bmod n_{j}, 0 \leqq i^{\prime} \leqq n_{j}-1$.

Now $D(f)=\delta(f)^{2}$ where

$$
\delta(f)=\prod_{\left(i_{1}, j_{1}\right)<\left(i_{2}, j_{2}\right)}\left(\sigma^{i_{1}} \beta_{j_{1}}-\sigma^{i_{2}} \beta_{j_{2}}\right) .
$$


If we apply $\sigma$ to $\delta(f)$, we get

$$
\sigma \delta(f)=\prod_{\left(i_{1} j_{1}\right)<\left(i_{2}, j_{2}\right)}\left(\sigma^{i_{1}+1} \beta_{j_{1}}-\sigma^{i_{2}+1} \beta_{j_{2}}\right) .
$$

The terms occurring in this product are the same as those in $\delta(f)$ except for sign. The term $\sigma^{i_{1}+1} \beta_{j_{1}}-\sigma^{i_{2}+1} \beta_{j_{2}}$ occurs in $\delta(f)$ and $\sigma \delta(f)$ with the same sign if and only if $\left(i_{1}+1, j_{1}\right)<\left(i_{2}+1, j_{2}\right)$. This is certainly the case if $j_{1}<j_{2}$ or if $j_{1}=j_{2}=j$ and $i_{2}<n_{j}-1$. However, if $j_{1}=j_{2}=j$ and $i_{2}=n_{j}-1$, then $\left(i_{2}+1, j\right)=(0, j)<\left(i_{1}+1, j\right)$. Therefore the total number of terms which occur with different signs in $\delta(f)$ and $\sigma \delta(f)$ is equal to the number of pairs $\left(\left(i_{1}, j\right),\left(n_{j}-1, j\right)\right)$ where $0 \leqq i_{1} \leqq n_{j}-2$. There are $n_{j}-1$ such pairs for each $j$. The total number is given by

$$
\sum_{j}\left(n_{j}-1\right)=n-r .
$$

This shows that $\sigma \delta(f)=(-1)^{n-r} \delta(f)$. Now $D(f)$ is a square in $F$ if and only if $\delta(f) \in F$, which is the case if and only if $\sigma \delta(f)=\delta(f)$. Therefore $D(f)$ is a square in $F$ if and only if $n \equiv r \bmod 2$.

CoRollary 1. Let $K$ be a finite field of odd characteristic. Let $g(x)$ be a polynomial over $K$ of degree $n$ with no repeated root. Let $r$ be the number of irreducible factors of $g(x)$ over $K$. Then $r \equiv n$ $\bmod 2$ if and only if $D(g)$ is a square in $K$.

Proof. We can assume that $g(x)$ is monic. Let $F$ be a p-adic field with residue class field $K$. Let $f(x)$ be a monic polynomial over $F$ with integral coefficients such that $\bar{f}(x)=g(x)$. Then $D(g)$ is $D(f)$ reduced $\bmod \mathfrak{p}$. Since $D(g) \neq 0, D(f)$ is a square in $F$ if and only if $D(g)$ is a square in $K$. This follows immediately from Hensel's lemma applied to the polynomial $x^{2}-D(f)$.

A more elementary proof of Corollory 1 can be obtained by repeating the proof of Theorem 1 using $K$ in place of $F$.

If $f(x)$ is irreducible over $K, r=1$ and so $n$ is odd if and only if $D(f)$ is a square in $K$. This is the theorem of Dickson mentioned above.

If $K$ has characteristic 2, the proof of Corollary 1 breaks down because $D(g)$ may be a square $\bmod \mathfrak{p}$ even though $D(f)$ is not a square. For example, 3 is a square $\bmod 2$ but not $\bmod 8$. In this case we need the following well-known result.

Lemma 1. Let a be a p-adic integer prime to $\mathfrak{p}$. Then $a$ is a $\mathfrak{p}$-adic square if and only if $a$ is a square $\bmod 4 \mathfrak{p}$.

Proof. Suppose $a \equiv b_{i}^{2} \bmod 4 \mathfrak{p}^{i}$. Then $a=b_{i}^{2}+4 c_{i}$ where $c_{i} \equiv 0$ 
$\bmod \mathfrak{p}^{i}$. Let $d_{i}=b_{i}^{-1} c_{i}$. Then $d_{i} \equiv 0 \bmod \mathfrak{p}^{i}$ since $b_{i}$ is prime to $\mathfrak{p}$, and $a=\left(b_{i}+2 d_{i}\right)^{2}-4 d_{i}^{2}$. Let $b_{i+1}=b_{i}+2 d_{i}$. Then $a \equiv b_{i+1}^{2} \bmod 4$ $\mathfrak{p}^{i+1}$ (in fact $\left.\bmod 4 \mathfrak{p}^{2 i}\right)$. The $b_{i}$ form a Cauchy sequence and $a=b^{2}$ where $b=\lim b_{i}$.

COROLlary 2. Let $f(x)$ be a monic polynomial of degree $n$ with integral coefficients over a p-adic field $F$. Assume that $\bar{f}(x)$ has no repeated root. Let $r$ be the number of irreducible factors of $\bar{f}(x)$ over the residue class field $K$ of $F$. Then $r \equiv n \bmod 2$ if and only if $D(f)$ is a square $\bmod 4 \mathfrak{p}$.

This follows immediately from Theorem 1 and Lemma 1. In applying Corollary 2 we are usually given $K$ and $\bar{f}(x)$ and choose any convenient $F$ and $f(x)$. For example, in case $K=G F(2)$, we get

CoRollary 3. Let $g(x)$ be a polynomial of degree $n$ over $G F(2)$ with no repeated root. Let $r$ be the number of irreducible factors of $g(x)$ over $G F(2)$. Let $f(x)$ be any monic polynomial over the 2-adic integers such that $\bar{f}(x)=g(x)$. Then $r \equiv n \bmod 2$ if and only if $D(f) \equiv 1 \bmod 8$.

This follows immediately from Corollary 2 and the fact that 1 is the only odd square $\bmod 8$. Note that $D(f) \equiv 1$ or $5 \bmod 8$ by Stickelberger's theorem.

ExAMPLe. Let $f(x)$ be a polynomial of degree $k$ over a finite field of characteristic 2 such that $f(0) \neq 0$. Let $g(x)=f(x)^{8}+x^{m}$ where $m$ is odd. Then $n=\operatorname{deg} g(x)=\max (8 k, m)$. Choose an appropriate $\mathfrak{p}$ adic field and a polynomial $F(x)$ of degree $k$ such that $f(x)=\bar{F}(x)$. Then $g(x)=\bar{G}(x)$ where $G(x)=F(x)^{8}+x^{m}$. Now $G^{\prime}(x) \equiv m x^{m-1} \bmod$ 8 so

$$
D(G) \equiv(-1)^{n(n-1) / 2} \Pi m \alpha_{i}^{m-1}(\bmod 8)
$$

Since $\Pi \alpha_{i}=(-1)^{n} G(0) \equiv f(0)^{8} \not \equiv 0 \bmod \mathfrak{p}, D(G) \not \equiv 0 \bmod \mathfrak{p}$ so $g(x)$ has no repeated root. Since $m$ is odd, $\Pi \alpha_{i}^{m-1}$ is a square. Thus $D(G)$ differs by a square factor from

$$
D^{\prime}=(-1)^{n(n-1) / 2} m^{n}
$$

If $n=8 k, D^{\prime}$ is a square. Thus $r \equiv n \equiv 0 \bmod 2$. Therefore $g(x)$ has an even number of factors and so is reducible. If $n=m, D^{\prime}$ differs by a square factor from $(-1)^{(m-1) / 2} m$. If $m \equiv \pm 3 \bmod 8,(-1)^{(m-1) / 2} m \equiv 5$ 
$\bmod 8$ and so $r \not \equiv n \equiv 1 \bmod 2$. Therefore $g(x)$ is reducible if $m \equiv \pm 3$ $\bmod 8$.

In particular, $x^{8 k}+x^{m}+1$ is reducible $\bmod 2$ if $m<8 k$. If $m>8 k$ it is reducible if $m \equiv \pm 3 \bmod 8$.

3. Quadratic reciprocity. The discriminant of the polynomial $x^{n}+a$ over any field is given by

$$
D\left(x^{n}+a\right)=(-1)^{n(n-1) / 2} \Pi n \alpha_{i}^{n-1}=(-1)^{n(n-1) / 2} n^{n} a^{n-1}
$$

because $\Pi \alpha_{i}=(-1)^{n} a$.

Consider in particular the polynomial $x^{p}-1=(x-1) \Phi_{p}(x)$ where $p$ is an odd prime. Its discriminant differs by a square factor from $(-1)^{(p-1) / 2} p$. Therefore $x^{p}-1$ has an odd number of factors over $G F(2)$ if and only if $p \equiv \pm 1 \bmod 8$. If $q \neq p$ is an odd prime, $x^{p}-1$ has an odd number of factors over $G F(q)$ if and only if $(-1)^{(p-1) / 2} p$ is a square $\bmod q$.

Now, if $\alpha$ is any root of $\Phi_{p}(x)$ over $G F(q), q \neq p, \alpha$ is a primitive $p$ th root of unity. Therefore $\alpha \in G F\left(q^{n}\right)$ if and only if $p \mid q^{n}-1$. Thus the degree of $\alpha$ (and hence of any irreducible factor of $\Phi_{p}(x)$ ) over $G F(q)$ is the order $n$ of $q \bmod p$. It follows that $x^{p}-1$ has $1+\varphi(p) / n$ factors over $G F(q)_{\rho}$

Since the multiplicative group $Z_{p}^{*}$ of integers $\bmod p$ is cyclic, $Z_{p}^{*}$ has a unique subgroup of index 2 which consists of all squares. Thus $q$ is a square mod $p$ if and only if it generates a subgroup of $Z_{p}^{*}$ of even index. This index, however, is just $\varphi(p) / n$, so $q$ is a square $\bmod$ $p$ if and only if $x^{p}-1$ has an odd number of factors over $G F(q)$. Comparing this with the results obtained from Theorem 1, we get

$$
\begin{aligned}
& \left(\frac{q}{p}\right)=\left(\frac{(-1)^{(p-1) / 2} p}{q}\right) \text { if } q \text { is odd } \\
& \left(\frac{2}{p}\right)=1 \text { if and only if } p \equiv \pm 1 \bmod 8 .
\end{aligned}
$$

These equations, together with the trivial formula

$$
\left(\frac{-1}{p}\right)=(-1)^{(p-1) / 2}
$$

constitute the quadratic reciprocity theorem.

4. Calculations. For the calculations made above, the discriminant could easily be found using the formula given in $\S 1$. However, for more complicated polynomials this method of finding the discriminant is very inefficient. In this section I will give a simpler method based on 
the Euclidean algorithm and use it to calculate the discriminant of any trinomial.

Let $f$ and $g$ be any polynomials over any field $F$. Let $g$ have roots $\beta_{1}, \cdots, \beta_{m}$, (counted with multiplicity), and leading coefficient $b$. Let $n$ be the degree of $f(x)$. Then the resultant of $f$ and $g$ is defined to be

$$
R(f, g)=b^{n} \prod_{j=1}^{m} f\left(\beta_{j}\right) .
$$

Clearly $R(f, g) \in F$ since it is a symmetric function in the roots of $g$. Comparing this definition with the formula for $D(f)$ given in $\S 1$ shows that if $f$ is monic

$$
D(f)=(-1)^{n(n-1) / 2} R\left(f^{\prime}, f\right) .
$$

The following properties of $R(f, g)$ are presumably well known, but I will include them for completeness.

LEMMA 2. (1) $R(g, f)=(-1)^{\operatorname{deg} g \cdot \operatorname{deg} g} R(f, g)$

(2) If $f=g q+r$,

$$
R(f, g)=b^{\operatorname{deg} f-\operatorname{deg} r} R(r, g)
$$

where $b$ is the leading coefficient of $g$.

(3) If $a$ and $b$ are constants not both $0, R(a, b)=1$.

(4) $R\left(f_{1} f_{2}, g\right)=R\left(f_{1}, g\right) R\left(f_{2}, g\right)$.

The proof is trivial.

CoRollaRY 4. (5) $R\left(f, g_{1} g_{2}\right)=R\left(f, g_{1}\right) R\left(f, g_{2}\right)$

(6) If $a$ is contant, $R(f, a)=a^{\operatorname{deg} f}=R(a, f)$

(7) $\quad R\left(f, x^{m}\right)=R(f, x)^{m}=f(0)^{m}$.

It follows from properties (1), (2), and (3) of Lemma 2 that we can compute $R(f, g)$ by applying the Euclidean algorithm to $f$ and $g$. This method of computation seems much easier in practice than the rather cumbersome determinant formula [5, Ch. 11, §71, 72.].

In order to compute the discriminant of a trinomial, it is first necessary to compute the resultant of two binomials.

LEMMA 3. Let $d=(r, s)$ be the greatest common divisor of $r$ and s. Let $r=d r_{1}, s=d s_{1}$. Then $R\left(x^{r}-\alpha, x^{s}-\beta\right)=(-1)^{s}\left[\alpha^{s_{1}}-\beta^{r_{1}}\right]^{a}$.

Proof. We first observe that if the result holds for a given pair $(r, s)$ it holds for $(s, r)$. This follows easily from Lemma 2 (1) using the fact $r s+s+d \equiv r \bmod 2$. 
Since the result is trivial for $s=0$, we can prove it by induction on $r+s$, assuming also $r \geqq s$ by the previous remark.

Now, dividing $x^{r}-\alpha$ by $x^{s}-\beta$ gives the remainder $\beta x^{r-s}-\alpha$. Thus we can apply Lemma 2 (2) and the result follows easily by induction. field.

It is now easy to find the discriminant of a trinomial over any

Theorem 2. Let $n>k>0$. Let $d=(n, k)$ and $n=n_{1} d, k=k_{1} d$. Then

$$
D\left(x^{n}+a x^{k}+b\right)=(-1)^{n(n-1) / 2} b^{k-1}\left[n^{n_{1}} b^{n_{1}-k_{1}}+(-1)^{n_{1}+1}(n-k)^{n_{1}-k_{1}} k^{k_{1}} a^{n_{1}}\right]^{a} .
$$

Proof. Consider the generic polynomial $f$ of degree $n$, multiply out

$$
D(f)=\prod_{i<j}\left(\alpha_{i}-\alpha_{j}\right)^{2}
$$

and express the resulting symmetric functions as integral polynomials in the coefficients of $f$. This gives an expression for $D(f)$ as a specific integral polynomial in the coefficients of $f$ and this expression is independent of the characteristic. In order to find the form of this polynomial, it is sufficient to do it in characteristic 0. For any polynomial $f$ of degree $n$,

$$
D(f)=(-1)^{n(n-1) / 2} R\left(f^{\prime}, f\right) .
$$

Therefore

$$
\begin{gathered}
D\left(x^{n}+a x^{k}+b\right)=(-1)^{n(n-1) / 2} R\left(n x^{n-1}+k a x^{k-1}, x^{n}+a x^{k}+b\right) \\
=(-1)^{n(n-1) / 2} n^{n} b^{k-1} R\left(x^{n}+a x^{k}+b, x^{n-k}+n^{-1} k a\right)
\end{gathered}
$$

using Lemma 2 (1), (4) and Corollary 4 (7)

Now, dividing $x^{n}+a x^{k}+b$ by $x^{n-k}+n^{-1} k a$ leaves the remainder $a\left(1-n^{-1} k\right) x^{k}+b$. Therefore the result follows from Lemma 2 (2) and Lemma 3.

As an application of Theorem 3, I will determine the parity of the number of factors of $x^{n}+x^{k}+1$ over $G F(2)$.

Corollary 5. Let $n>k>0$. Assume exactly one of $n, k$ is odd. Then $x^{n}+x^{k}+1$ has an even number of factors (and hence is reducible) over $G F(2)$ in the following cases.

(a) $n$ is even, $k$ is odd, $n \neq 2 k$ and $n k / 2 \equiv 0$ or $1 \bmod 4$

(b) $n$ is odd, $k$ is even, $k \nmid 2 n$, and $n \equiv \pm 3 \bmod 8$

(c) $n$ is odd, $k$ is even, $k \mid 2 n$, and $n \equiv \pm 1 \bmod 8$

In all other cases $x^{n}+x+1$ has an odd number of factors over $G F(2)$. 
The case where $n$ and $k$ are both odd can be reduced to the case $k$ even by considering $x^{n}+x^{n-k}+1$ which has the same number of irreducible factors as $x^{n}+x^{k}+1$.

To prove Corollary 5 we regard $x^{n}+x^{k}+1$ as a polynomial over the 2-adic integers, compute its discriminant by Theorem 2, and apply Corollary 3.

Note that the fact that some trinomial $x^{n}+x^{k}+1$ has an odd number of factors does not imply that it is irreducible. For example, we may consider the trinomial $x^{2 k}+x^{k}+1$ with $k$ odd. In a number of cases, including this one, the reducibility or irreducibility of $x^{n}+x^{k}$ +1 can be decided by using the results of [1, Ch. V, §9]. Recall that an irreducible polynomial $f(x)$ over a finite field is said to belong to the exponent $e$ if $e$ is the least integer such that $f(x) \mid x^{e}-1$. In other words, $e$ is the order of a root of $f(x)$.

If $f(x)$ is irreducible of degree $n$ and exponent $e$ over $G F(q)$, it follows from [1, Ch. V, Th. 18] that $f\left(x^{s}\right)$ is irreducible over $G F(q)$ if and only if every prime $p$ dividing $s$ also divides $e$ but does not divide $\left(q^{n}-1\right) / e$ and, in addition, $4 \nmid s$ if $q^{n} \equiv-1 \bmod 4$.

In particular $x^{2 k}+x^{k}+1$ is irreducible over $G F(2)$ if and only if $k$ is a power of 3 and $x^{4 k}+x^{k}+1$ is irreducible over $G F(2)$ if and only if $k=3^{r} 5^{s}$.

Some other cases can be disposed of by observing that if $x^{r}+x^{s}+1$ divides $x^{e}+1$ then $x^{r}+x^{s}+1$ divides $x^{n}+x^{k}+1$ if $n \equiv r, k \equiv s \bmod$ $e$. For emample, $x^{2}+x+1$ divides $x^{n}+x^{k}+1$ if $n \equiv 2, k \equiv 1 \bmod 3$ or if $n \equiv 1, k \equiv 2 \bmod 3$.

REMARK. I. Kaplansky points out that Theorem 1 can be reformulated so as to avoid the use of $\mathfrak{p}$-adic numbers by considering the positive and negative terms in $\Pi\left(\alpha_{i}-\alpha_{j}\right)$. These are polynomials in the $\alpha_{i}$ which also make sense in characteristic 2. This yields an elementary form of the theorem but one which is hard to apply because of the difficulty in computation.

\section{REFERENCES}

1. A. A. Albert, Fundamental Concepts of Higher Algebra, University of Chicago Press, 1956.

2. On certain trinomial equations in finite fields, Ann. of Math., 66 (1957), (1957), 170-178.

3. E. Artin, Theory of Algebraic Numbers, Gottingen, 1959.

4. H. S. Vandiver, On trinomial equations in a finite field, Proc. Nat. Acad. Sci. U.S.A.

40 (1954), 1008-1010.

5. B. L. van der Waerden, Moderne Algebra, Julius Springer, Berlin, 1931. 


\title{
PACIFIC JOURNAL OF MATHEMATICS
}

\author{
EDITORS
}

RalPh S. Phillips

Stanford University

Stanford, California

M. G. Arsove

University of Washington

Seattle 5 , Washington
A. L. Whiteman

University of Southern California Los Angeles 7, California

LOWell J. PAIGE

Unıversity of California

Los Angeles 24, California

\section{ASSOCIATE EDITORS}
E. F. BECKENBACH
D. DERRY
H. L. ROYDEN
E. G. STRAUS
T. M. CHERRY
M. OHTSUKA
E. SPANIER
F. WOLF

\section{SUPPORTING INSTITUTIONS}

\author{
UNIVERSITY OF BRITISH COLUMBIA \\ CALIFORNIA INSTITUTE OF TECHNOLOGY \\ UNIVERSITY OF CALIFORNIA \\ MONTANA STATE UNIVERSITY \\ UNIVERSITY OF NEVADA \\ NEW MEXICO STATE UNIVERSITY \\ OREGON STATE UNIVERSITY \\ UNIVERSITY OF OREGON \\ OSAKA UNIVERSITY \\ UNIVERSITY OF SOUTHERN CALIFORNIA
}

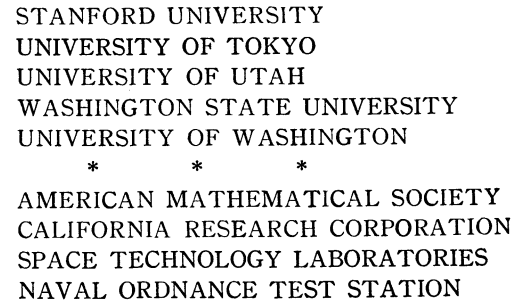

Mathematical papers intended for publication in the Pacific Journal of Mathematıcs should be typewritten (double spaced), and the author should keep a complete copy. Manuscripts may be sent to any one of the four editors. All other communications to the editors should be addressed to the managing editor, L. J. Paige at the University of California, Los Angeles 24, California.

50 reprints per author of each article are furnıshed free of charge; additional copies may be obtained at cost in multiples of 50 .

The Pacific Journal of Mathematics is published quarterly, in March, June, September, and December. Effective with Volume 13 the price per volume (4 numbers) is $\$ 18.00$; single issues, $\$ 5.00$. Special price for current issues to individual faculty members of supporting institutions and to individual members of the American Mathematical Society: $\$ 8.00$ per volume; single issues $\$ 2.50$. Back numbers are available.

Subscriptions, orders for back numbers, and changes of address should be sent to Pacific Journal of Mathematics, 103 Highland Boulevard, Berkeley 8, California.

Printed at Kokusai Bunken Insatsusha (International Academic Printing Co., Ltd.), No. 6 , 2-chome, Fujimi-cho, Chiyoda-ku, Tokyo, Japan.

PUBLISHED BY PACIFIC JOURNAL OF MATHEMATICS, A NON-PROFIT CORPORATION

The Supporting Institutions listed above contribute to the cost of publication of this Journal, but they are not owners or publishers and have no responsibility for its content or policies. 


\section{Pacific Journal of Mathematics}

\section{Vol. 12, No. $3 \quad$ March, 1962}

Alfred Aeppli, Some exact sequences in cohomology theory for Kähler

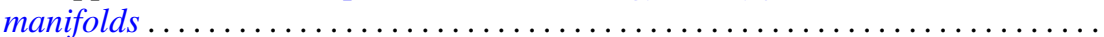

Paul Richard Beesack, On the Green's function of an N-point boundary value

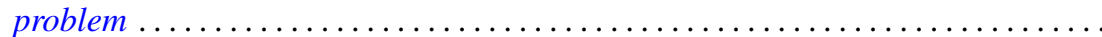

James Robert Boen, On p-automorphic p-groups....

James Robert Boen, Oscar S. Rothaus and John Griggs Thompson, Further results

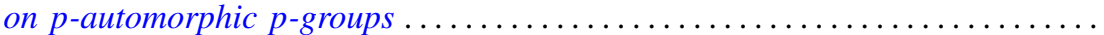

James Henry Bramble and Lawrence Edward Payne, Bounds in the Neumann problem for second order uniformly elliptic operators ..................

Chen Chung Chang and H. Jerome (Howard) Keisler, Applications of ultraproducts of pairs of cardinals to the theory of models .........................

Stephen Urban Chase, On direct sums and products of modules ................

Paul Civin, Annihilators in the second conjugate algebra of a group algebra .......

J. H. Curtiss, Polynomial interpolation in points equidistributed on the unit

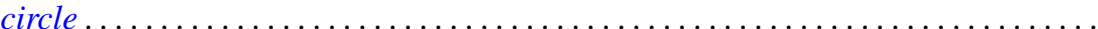

Marion K. Fort, Jr., Homogeneity of infinite products of manifolds with

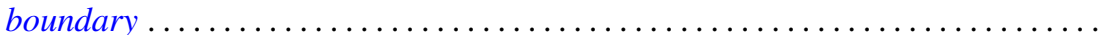

James G. Glimm, Families of induced representations . . . . . . . . . . . . . . .

Daniel E. Gorenstein, Reuben Sandler and William H. Mills, On almost-commuting

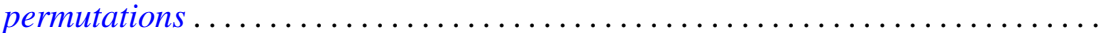

Vincent C. Harris and M. V. Subba Rao, Congruence properties of $\sigma_{r}(N) \ldots \ldots \ldots$

Harry Hochstadt, Fourier series with linearly dependent coefficients . . . . . . . . . . 925

Kenneth Myron Hoffman and John Wermer, A characterization of $C(X)$. .

Robert Weldon Hunt, The behavior of solutions of ordinary, self-adjoint differential equations of arbitrary even order...

Edward Takashi Kobayashi, A remark on the Nijenhuis tensor

David London, On the zeros of the solutions of $w^{\prime \prime}(z)+p(z) w(z)=0$

Gerald R. Mac Lane and Frank Beall Ryan, On the radial limits of Blaschke products...

T. M. MacRobert, Evaluation of an E-function when three of its upper parameters

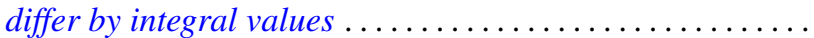

Robert W. McKelvey, The spectra of minimal self-adjoint extensions of a symmetric operator

Adegoke Olubummo, Operators of finite rank in a reflexive Banach space. .

David Alexander Pope, On the approximation of function spaces in the calculus of variations

Bernard W. Roos and Ward C. Sangren, Three spectral theorems for a pair of singular first-order differential equations...............

Arthur Argyle Sagle, Simple Malcev algebras over fields of characteristic zero .

S. C. Tang, Some theorems on the ratio of empirical distribution to the theoretical distribution

Robert Charles Thompson, Normal matrices and the normal basis in abelian number fields.

Howard Gregory Tucker, Absolute continuity of infinitely divisible distributions ... 